\title{
The Influence of Hepatocyte Growth Factor During Outer Segment Phagocytosis by Retinal Pigment Epithelium
}

\author{
J. F. Blaize*, J. Tachjadi*, W. J. L’Amoreaux ${ }^{* *}$ \\ The College of Staten Island (City University of New York) \\ 2800 Victory Blvd. Staten Island, NY. 10314. \\ *Graduate Program in Biology - Neuroscience, The City University of New York Graduate \\ Center, New York, NY 10016 \\ ${ }^{* *}$ Department of Biology and Advanced Imaging Facility, City University of New York, College \\ of Staten Island, Staten Island, NY 10314
}

Inhibition of outer segment (OS) processing by retinal pigment epithelium (RPE) has been linked to photoreceptor injury and retinopathy onset. Sub-retinal clearance by RPE is facilitated by specialized phagocytosis featuring both RPE-specific and traditional $\mathrm{FC} \gamma \mathrm{R}$ mediated signaling cascades. As a result of this combinatory approach, RPE are capable of internalizing both specific and non-specific external targets alike. The discovery that lack of c-Met signaling results in impairment of phagocytosis in alveolar and hepatocyte macrophages [1] suggests cMet's role as modulator of this activity in post-mitotic cells secreting HGF. Since activated PI3K has been identified as an activator of Rac1during FC $\gamma \mathrm{R}$ mediated phagocytosis, we hypothesize that c-Met activation by HGF and subsequent PI3K activation is capable of mediating OS clearance by RPE.

To test our hypotheses, cultured ARPE-19 cells were grown to 70\% confluence, then serum starved for $24 \mathrm{hr}$. Post starvation, cells were exposed to various concentrations of HGF for $24 \mathrm{hr}$ before fixation with $2.5 \%$ paraformaldehyde and .5\% glutaraldehyde. Cells were then prepared for immunohistochemistry for receptor expression (non-phosphorylated and phosphorylated forms), focal adhesion kinase (FAK) and binding of fluorescently-labeled E. coli. Intensity values suggest that ARPE-19 respond maximally to concentrations of $25 \mathrm{ng} / \mathrm{ml}$ of HGF when compared to controls (Fig 1). While phosphorylated c-Met was not significantly altered (Fig 2) at $24 \mathrm{hr}$, this may be attributed to the transient expression of phospho-c-Met following activation by HGF. Our findings suggest that RPE respond to increases of exogenous HGF concentrations by up-regulating its receptor and subsequent second messengers systems. In addition, our data show a significant increase of fluorescently labeled E. coli (Fig 3). Taken together, these findings suggest that RTK cross-talk initiated by c-Met activation may be sufficient in mediating general uptake of external debris by RPE. Future studies including RPE challenge with fluorescently labeled OS during peak c-Met phosphorylation evoked by increased HGF exposure will provide evidence for HGF's role as a mediator of specialized phagocytosis of OS.

\section{References}

[1] C.G. Huh et al., Proceedings of the National Academy of Science in the United States of America. 13 (2004) 101.

[2] Support for this project comes from PSC-CUNY and the LS-AMP program of CUNY. 


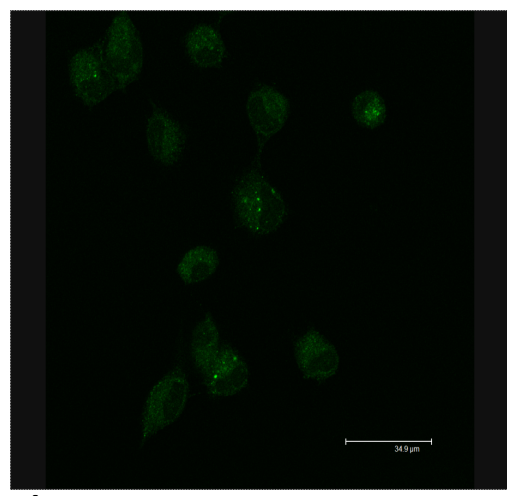

A

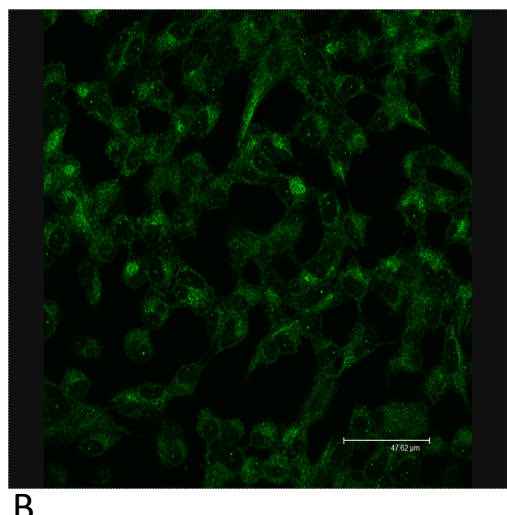

B

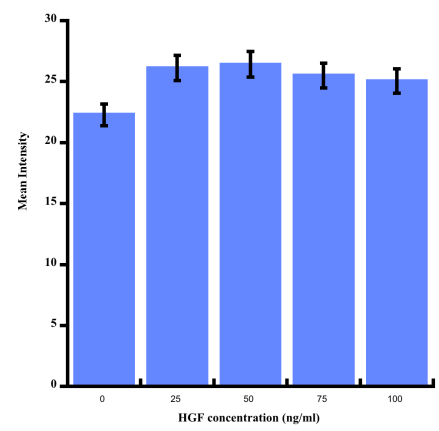

Figure 1 A \& B illustrate the up-regulation of c-Met in ARPE-19 when exposed to increasing concentrations of HGF. Statistical analyses reveal that all concentrations $\geq 25 \mathrm{ng} / \mathrm{ml}$ are significantly higher than controls, but concentrations higher than $25 \mathrm{ng} / \mathrm{ml}$ do not increase c-Met expression.

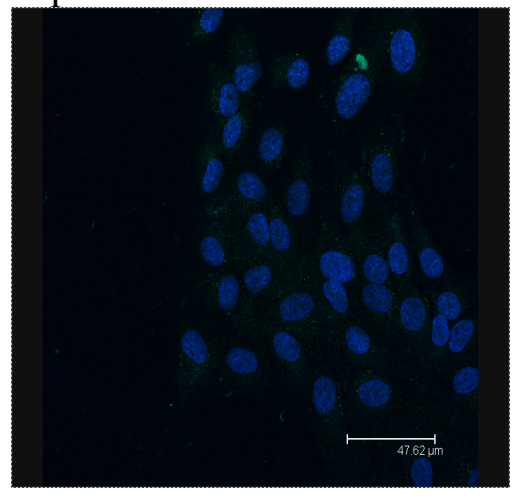

A

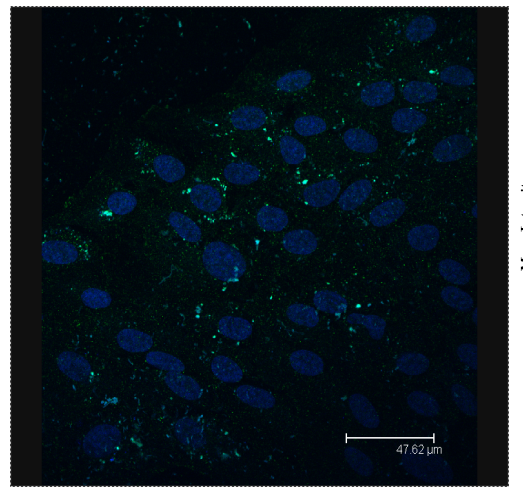

B

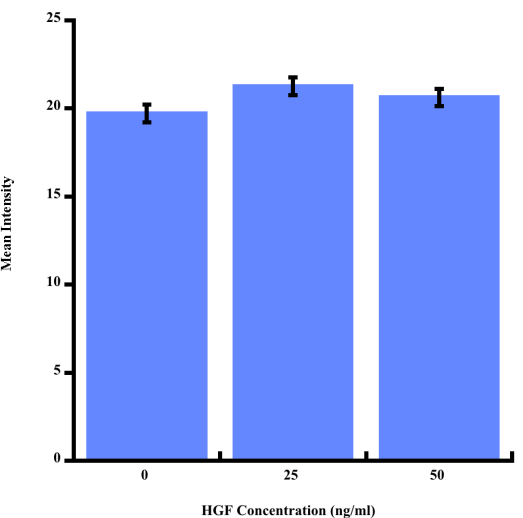

tration $(\mathrm{ng} / \mathrm{n})$

Figure 2 A \& B demonstrate that while cMet is up-regulated with HGF treatment, the phosphorylated cMet is not at $24 \mathrm{hr}$. This is likely due to both the constitutive expression of the protein and lack of HGF activation at $24 \mathrm{hr}$.

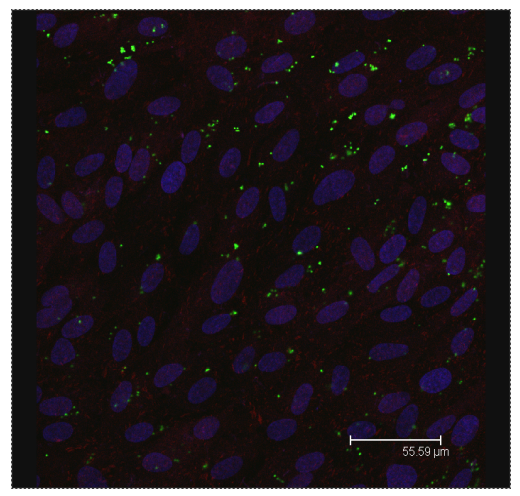

A
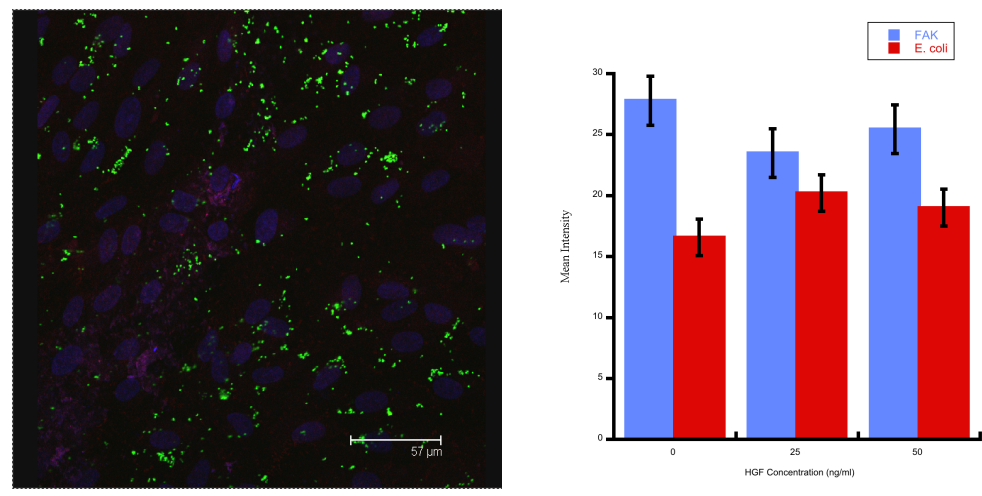

B

Figure $3 \mathrm{~A} \& \mathrm{~B}$ show an increase in extracellular target binding of fluorescently labeled $E$. coli by ARPE-19 treated with 25ng/ml HGF. Interestingly, total expression of FAK, a key mediator of specialized phagocytosis, is reduced after HGF treatment. 